\title{
Application of mind evolution Elman neural network model in gearbox fault diagnosis
}

Wang Hai jun,Jin Tao,Lin Zhi wei

Ordos Institute Of Technology, Inner Mongolia Ordos, 017000

\begin{abstract}
Gearbox as an important component of the power system of agricultural machinery, plays a vital role in the normal operation of agricultural equipment. At present, the diagnosis of gearbox fault is mainly based on expert experience, and the accuracy is not guaranteed. In view of the achievements of Elman neural network in fault diagnosis, Elman neural network is used as the basic model of gearbox fault diagnosis. Considering that the Elman neural network is sensitive to the initial weight threshold and easy to fall into the local minimum, the mind evolution algorithm is introduced into the parameter optimization of the Elman neural network fault diagnosis model. Experimental results show that this assumption is successful, and the new model not only reduces diagnostic errors but also improves stability.
\end{abstract}

\section{Introduction}

The gearbox as the main propulsion device of power system of all kinds of agricultural machinery and equipment to the tractor as the representative, is the core component of agricultural machinery power transmission gearbox, once the failure of mechanical system will inevitably lead to paralysis, lead to the agricultural machinery lost power, causing economic losses and even lead to accidents occurred. Studies have shown that the mechanical failure rate represented by a gearbox occupies all the 50\% 70\% except the engine. Therefore, the early diagnosis of agricultural machinery gearbox fault is of great significance to guarantee the power system of agricultural machinery. In the non disassembly condition, the traditional gearbox fault diagnosis often depends on the expert experience to judge. However, because the gearbox fault diagnosis model and feature vector is a very complex nonlinear relationship, the expert experience can not completely solve the gear box fault diagnosis problem ${ }^{[1]}$.

The existing studies have shown that Elman neural network has very good application effect in fault diagnosis, Elman neural network is improved based on BP neural network with feedback, local structure, ability to handle dynamic information better. But because the Elman neural network also uses the BP neural network algorithm to correct the weight, there are some shortcomings, such as slow learning speed and easy to fall into local minimum ${ }^{[2,3]}$. Therefore, the emergence of a large number of Elman neural network based on swarm intelligent algorithm improved algorithm model, Huang Yuchun ${ }^{[4]}$ using genetic algorithm to optimize Elman neural network to forecast the thermal error achieved good results, Chen $\mathrm{Fafa}^{[5]}$ by optimization of Elman neural network in fault diagnosis of rotating machinery can significantly improve the accuracy of fault diagnosis of rolling bearing immune genetic algorithm. Can be seen from these studies that the swarm intelligence algorithm into the Elman neural network model can also improve operation efficiency.

In view of this, the Mind Evolutionary Algorithm (MEA) is introduced into the Elman model optimization, and the gearbox fault diagnosis model based on the thought evolution Elman neural network algorithm is constructed. The experimental results show that compared with the neural network model of Elman algorithm and BP algorithm based on mind evolutionary, the diagnosis model of mind evolutionary Elman neural network algorithm is superior to the former in the stability and accuracy of diagnosis based on operation.

\section{Elman neural network}

Elman neural network is proposed by J.L.Elman in 1990 which is a dynamic feedback network, compared with the traditional BP neural network, Elman neural network adds a layer of memory unit based on BP network, called undertake layer, through the internal storage of intermediate state, which can output to the hidden layer unit before the moment value of memory, so the network has a dynamic memory function. The working process of the Elman neural network is first initialized to the neural network the right threshold, the signal from the input layer to the hidden layer input, after weighting computation, the output results to the hidden layer and the output layer, the results are calculated, calculated results to undertake temporarily stored in the layer, and the calculated layer to undertake the output feedback back to the hidden layer re calculate the output output error, the final calculation of two times the output of the system, update the right threshold for next calculation, the calculation model is shown as the following formula ${ }^{[6]}$.

$$
\begin{aligned}
& x(k)=f\left(W^{2} x_{c}(k)+W^{1} u(k-1)\right) \\
& x_{c}(k)=\alpha x_{c}(k-1)+x(k-1) \\
& y(k)=g\left(W^{3} x(k)\right)
\end{aligned}
$$

Among them, $x(k)$ and $x_{c}(k)$ represent the output of the hidden layer and the receiving layer, respectively, $y(k)$ is the output of the output layer, $u$ is the input vector, $W^{1}$ is the weight of the input layer to the hidden layer, $W^{2}$ is the weight of the hidden layer to the receiving layer, $W^{3}$ is the connection weight of the hidden layer to the output layer, $f(\cdot)$ and $g(\cdot)$ are the transfer functions of the hidden layer and the output layer, respectively, $0 \leq \alpha \leq 1$ is a feedback gainfactor for self connection.

\footnotetext{
* Corresponding author:wanghaijun11249@126.com
} 
Let $y_{r}(k)$ indicate the actual output of step $\mathrm{k}$ of the system, Define the error objective function, as shown in formula (4)

$$
E(k)=(\bar{y}(k)-y(k))^{T}(\bar{y}(k)-y(k)) / 2
$$

Then the dynamic learning algorithm of Elman neural network can be summarized as follows

$$
\begin{aligned}
& \Delta w_{i j}^{3}=\eta\left(y_{i}(k)-y_{i}(k)\right) g_{i}^{\prime}(\cdot) x_{j}(k) \\
& (i=1,2, \cdots, m ; j=1,2, \cdots, n) \\
& \Delta w_{j q}^{2}=\eta \sum_{i=1}^{m}\left(\overline{y_{i}}(k)-y_{i}(k)\right) g_{i}^{\prime}(\cdot) w_{i j}^{3} f_{j}^{\prime}(\cdot) u_{q}(k-1) \\
& (j=1,2, \cdots, n ; q=1,2, \cdots, r) \\
& \Delta w_{j l}^{3}=\eta \sum_{i=1}^{m}\left(y_{i}(k)-y_{i}(k)\right) g_{i}^{\prime}(\cdot) w_{i j}^{3} \frac{\partial x_{j}(k)}{\partial w_{j l}^{1}} \\
& (j=1,2, \cdots, n ; l=1,2, \cdots, n) \\
& \frac{\partial x_{j}(k)}{\partial w_{j l}^{1}}=f_{j}^{\prime}(\cdot) x_{l}(k-1)+\alpha \frac{\partial x_{j}(k)}{\partial w_{j l}^{1}}
\end{aligned}
$$

\section{Mind evolution Elman neural network algorithm design}

\subsection{Algorithm core idea}

The theoretical basis of the Elman neural network algorithm for mind evolution is the effective combination of the two algorithms through parameter transfer. The mind evolution algorithm takes the initial weight threshold parameter of the Elman algorithm as a solution group, which is called the initial population. In the process of evolution, the initial species group is divided into several sub groups, which are divided into two groups: the dominant group and the temporary group. Each sub group has high fitness value as the center, and generates $M$ individuals according to certain rules. Within the sub population, individual competition becomes a winner, called a seed of a sub population. It is the basis for the next step of evolution. This process is called local convergence. The winners of each sub group compete with each other, according to the fitness value, the survival of the fittest, and find a new point of proportion in the solution space. This process is called "overall alienation". The convergence of operation to achieve the individual internal sub groups competing in the local domain, complete local search operation to achieve the global competition, alienation, guarantee group global search capability, reduce the probability of falling into local optimal, make the population toward the global optimum evolution ${ }^{[7,8]}$. After several generations of the convergence process of alienation eventually survived the initial solution, the optimal threshold of Elman algorithm is to find the right, then the threshold parameter to Elman algorithm model are two optimization, find the best solution. Figure 1 is the whole process of the algorithm combining the mind evolution algorithm with the Elman algorithm.

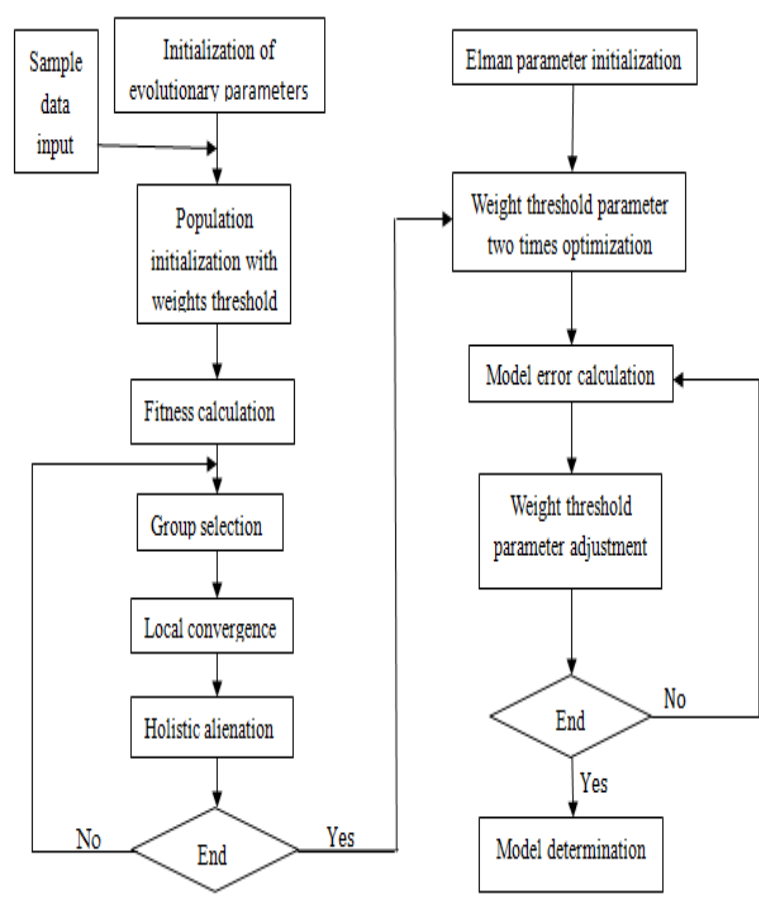

Figure 1 Mind evolution, Elman algorithm, flow chart

\subsection{Algorithm execution step design}

Step1 Population initialization-Using mind evolutionary algorithm to initialize population, let $W 1$ be the input layer to the hidden layer weight $W 2$ is the weight from the hidden layer to the output layer, $W_{3}$ is the weight of the hidden layer to the receiving layer、 $B 1$ is the threshold of the input layer to the hidden layer, $B 2$ is the threshold from the hidden layer to the output layer.Because the Elman network is based on the BP gradient descent algorithm, therefore, only $W 1, W 2, B 1$ and $B 2$ are encoded and optimized here, $W 3$ is randomly generated by the system. Figure 2 shows the basic body diagram of the Elman neural network used in this article, where each individual in the initial population is shown in the following formula.

$\{W 1, W 2, B 1, B 2\}$

In the group, the nodes in each individual are randomly generated in $[-1,1]$ by the probability shown by formula (10).

$P_{\mathrm{ij}}=1 /(S+1)$

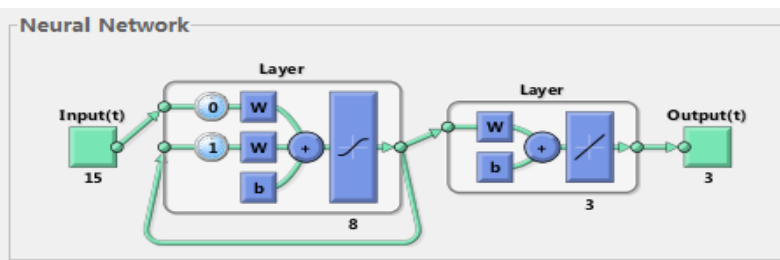

Figure 2 the basic structure of the Elman model

Step2 Construction of fitness function-The fitness value calculation process and external information interaction in the whole operation process of individual evolutionary algorithms, evolutionary function is the only standard evolutionary basis, 
therefore evolutionary function selection will directly affect the operation of the final result of the algorithm. The algorithm uses the reciprocal of the error squared between the actual value and the desired output of the gearbox fault diagnosis model and the reciprocal of the $\mathrm{SE}$ as the basis for the algorithm, This can effectively integrate the Elman algorithm with the evaluation criteria of the mind evolutionary algorithm, Evolutionary functions such as equation (11) are shown:

$F$ val $=1 / S E=1 / \sum_{i=1}^{n}\left(y_{i}-t_{i}\right)$

Fval is the fitness value in the formula, $y_{i}$ is the expected output of the Elman model, $t_{i}$ is for actual output.

Step3 Group selection-Group selection is the key step for Population Division in the process of mind evolution, In this paper, the population size is set to psize $=100$, the winner group size bsize $=10$, the provisional subgroup tpsize $=10$. According to the formula (11), the individual fitness value of the population was calculated, and sort them from high to low, select bsize+tsize individuals as the winning group. Finally, the whole population is divided into bsize dominant sub groups and tsize temporary sub groups, each of which is $S G$ in size.

$S G=$ psize $/($ bsize + tpsize $)$

Step4 Individual convergence-After selecting the superior, a number of subgroups with normal size of $N(\mu, \Sigma)$ are generated with $S G$ size, $\mu$ and $\sum$ are central vectors and covariance matrices of normal distribution.$\mu$ is the individual location with the highest fitness value, that is the solution of the problem we ask. If $\sum$ is diagonal matrix, this means that individuals in a subgroup are independent of each other. After $i$ generation operation, $\left\{\sigma_{i d}\right\}$ is a non-zero individual in $\sum$, among them, $d=0,1, \ldots, S$ represents the dimension of the solution space. Each one-dimensional variance of normal distribution is calculated as follows.

$\sigma_{0 d}=\left(H_{d}-L_{d}\right) / 10$

In the formula $H_{d}$ and $L_{d}$ denote the upper and lower bounds of the dimension respectively. In the iterative process, the highest score in the variance is the optimal solution. The formula for the variance of any two generations is as follows ${ }^{[9]}$.

$$
\sigma_{(i+1) d}=c_{1} \sigma_{i d}+c_{2} \delta
$$

Where $c_{1}$ and $c_{2}$ are inter generational adjustment coefficient. $\delta$ stands for the distance between the two generations. In this article, set $c_{1}=1, c_{2}=0.5$. set $\delta=\operatorname{rand}(1, S) * 2-1$ to increase the gap between the two generations and increase search speed.

Step5 Holistic alienation-After convergence, all groups changed, Therefore, the individuals in the swarm need to re calculate the order of fitness calculation in accordance with formula (11), individual fitness values of the better and temporary groups. The fitness value of the fitness group with the highest fitness value is $T_{\max }, \mathrm{A}$ set consisting of individuals with fitness values greater than $T_{\max }$ in the temporary group is denoted as
Temp $=\left\{t_{1}, t_{2}, \cdots, t_{p}\right\}$,Find the fitness in the winner group, and the last $\mathrm{p}$ individuals are the set Best $=\left\{b_{1}, b_{2}, \cdots, b_{p}\right\}$. Replacing the Best with the Temp constitutes a new fitness group with the highest fitness value, and discards the Best.At the same time, a new set $T e m p^{*}$ is generated randomly in the solution space, Temp $^{*}=\left\{t_{1}^{*}, t_{2}^{*}, \cdots, t_{p}^{*}\right\}$, and it was added to the temporary sub group to form a new temporary subgroup [10].

Step6 Iteration stop-The maximum iteration number is chosen as the convergence condition of the algorithm. If the convergence condition is not satisfied, the Step4 is returned to a new round of convergence and dissimilation operations until the iteration is stopped. The initial solution obtained by the optimization of the mind evolution algorithm is decoded, and then the Elman neural network loaded with the decoding value is trained and studied to form a diagnosis model.

\section{4 algorithm simulation}

\section{1 sample selection of input and output}

Most of the fault gear box are caused by gear fault, fault in the gear box parts, gear failure accounted for the largest proportion, accounting for about $60 \%$ of the time, bearing, so the emphasis of gearbox condition monitoring and fault diagnosis of gear fault diagnosis. This paper takes a tractor gearbox as the object of study, in the fault feature extraction, the amplitude $A_{i, j 1}, A_{i, j 2}$ and $A_{i, j 3}$ are selected in the frequency domain characteristic signal at 2, 4 and 6 files in the frequency band family $f_{s} \pm n f_{z}$ of the 1,2 and 3 axes, where $f_{s}$ represents the gear meshing frequency, and $f_{z}$ is the frequency of the shaft, $n=1,2,3, i=2,4,6$ stands for stalls, and $j=1,2,3$ stands for serial numbers of axes, because in the 2 axis and 3 axis two of gear meshing, so with 1 and 2 respectively represent the two meshing frequency. In this way, the state of the gear forms a vector combination of 15 eigenvalues $\left(A_{1,2}, A_{1,4}, A_{1,6}, A_{2,21}, A_{2,22}\right.$, $\left.A_{2,41}, A_{2,42}, A_{2,61}, A_{2,62}, A_{3,21}, A_{3,22}, A_{3,41}, A_{3,42}, A_{3,61}, A_{3,62}\right)$ which is used as the model input variable. Considering the Elman neural network model used in this paper to activate the function tansig $(x)=2 /(1+\exp (-2 x)-1)$ and purelin $(x)=x$, comparison of the sensitivity of these two functions of the input data, the input data are normalized to table 1 for the input data after normalization. As for the output node, this study only studies the 3 states of the gear, so the corresponding eigenvalue of Table 2 is used as the output.

Table 1 input values for training samples

\begin{tabular}{|c|c|c|c|c|}
\hline Enter Sample & 1 & 2 & $\ldots$ & 9 \\
\hline$A_{1,2}$ & 0.2286 & 0.2603 & $\ldots$ & 0.3171 \\
\hline$A_{1,4}$ & 0.1292 & 0.1715 & $\ldots$ & 0.1531 \\
\hline
\end{tabular}




\begin{tabular}{|c|c|c|c|c|}
\hline$:$ & $:$ & $:$ & $\ldots$ & $:$ \\
\hline$A_{3,62}$ & 0.1828 & 0.0909 & $\ldots$ & 0.1125 \\
\hline
\end{tabular}

Table 2sample output results

\begin{tabular}{|l|c|}
\hline Gear state & Output result \\
\hline Trouble free & $(1,0,0)$ \\
\hline Root crack & $(0,1,0)$ \\
\hline Broken teeth & $(0,0,1)$ \\
\hline
\end{tabular}

Set the network training target of 0.0001 , the learning coefficient is 0.1 , Training times is 100 . The hidden layer nodes are represented by formula (15) as the basic standard. After repeated experiments, they are determined as $15-8-3$, table 3 is the network error corresponding to different hidden layer nodes.

$$
\text { mid }=\sqrt{\text { input }+ \text { output }}+\alpha \quad \alpha=1,2, \ldots, 10
$$

Table 3 the corresponding error values of different hidden layer nodes

\begin{tabular}{|c|c|c|c|c|c|}
\hline number & 5 & 6 & 7 & 8 & 9 \\
\hline error & 0.0375 & 0.0330 & 0.0259 & 0.0217 & 0.0248 \\
\hline number & 10 & 11 & 12 & 13 & 14 \\
\hline error & 0.0266 & 0.0314 & 0.0329 & 0.0278 & 0.0332 \\
\hline
\end{tabular}

Since the Elman neural network is still essentially BP neural network, therefore, the corresponding training algorithm is consistent, a large number of studies have shown that the traingd algorithm based on gradient descent is slow in convergence and often fails to converge. Thus, a large number of improved algorithms appear, among which the Levenberg-Marquard (trainlm) algorithm is the most typical.Compared with other improved algorithms, faster convergence speed and higher efficiency with trainlm, figure 3 for the same problem, several BP algorithm performance comparison, can be seen from the figure, the trainlm algorithm is more efficient, therefore, trainlm algorithm is used as the Elman model training algorithm.

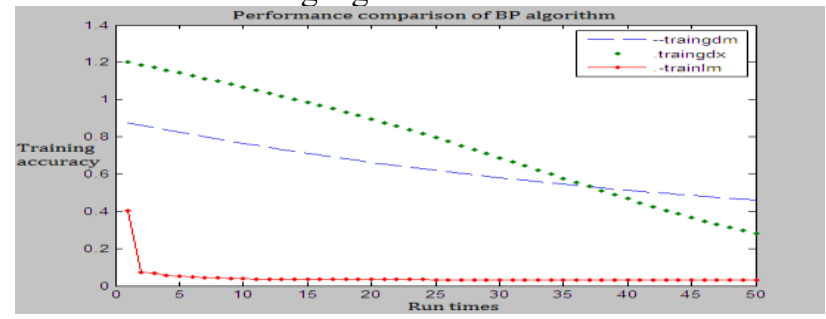

Figure 3 BP training result of improved algorithm

\subsection{Result analysis}

In view of the influence of initial parameters randomization of Elman model on the results, The Elman neural network model and the mind evolution BP model and the mind evolution Elman neural network model are used to diagnose the gear box faults respectively, and each runs 10 times. The final results are given in Table 4, and it can be seen from the table that the diagnostic error of the MEA-Elman neural network model is reduced by 0.0059 and 0.0112 compared to the MEA-BP model and the Elman model,
Compared with the MEA-Elman neural network model, the MEA-BP neural network model does not reduce much error. But because the running stability of the MEA-Elman neural network model than the other two models have improved, so the fault diagnosis rate compared with the other two models were increased by $6.6 \%$ and $16.6 \%$, we can see that the MEA-Elman neural network model in fault diagnosis accuracy is higher, the stability is better.

Table 4 Comparison of different model results based on different algorithms

\begin{tabular}{|l|l|c|}
\hline Model type & MSE & Fault diagnosis rate \\
\hline Elman & 0.0935 & $66.7 \%$ \\
\hline MEA-BP & 0.0882 & $76.7 \%$ \\
\hline MEA-Elman & 0.0823 & $83.3 \%$ \\
\hline
\end{tabular}

\section{Conclusion}

The mind evolutionary algorithm to Elman neural network model parameter optimization, search ability to optimize the Elman neural network weight and threshold parameters by using its powerful global, and build on the gearbox mind evolution model of Elman neural network fault diagnosis model based on. The experimental results show that this assumption is successful, and the Elman neural network, which introduces the mind evolution algorithm, can well overcome the sensitivity of the Elman neural network to the initial weight threshold and is easy to fall into the local minimum. Therefore, the diagnosis model not only has higher prediction accuracy, but also improves the stability, and proposes a feasible research method for agricultural machinery fault diagnosis research.

\section{Acknowledgements}

This work has been supported by Scientific research project of Inner Mongolia higher education institutions NJZY16382.

\section{Reference}

1 Song Chong Zhi, Wu Yu Guo, Wang Lu.Research on fault diagnosis of engine gearbox based on modified Elman neural network[J]Chinese Journal of Scientific Instrument, 2008,29(7):1414-1417

2 Zheng Min,Ren Fang, Yang Zhao jian. Study on the Load Identification of the Rotor System With SelfAdaptive BP Neural Networks[J] Machinery Design \& Manufacture, 2016,(6):85-89

3 Han Shu hua,Hou Xue yuan LI Wen qing. Study on Parameters Optimization of Injection Molding Based on Modified Particle Swarm Optimized Back Propagation Neural Network[J] Machinery Design \& Manufacture, 2015,(10):98-102

4 Huang Yu chun,Tian Jian ping,Yang Hai li. Thermal Error Modeling for Machine Tool Based on Genetic Algorithm Optimization Elman Neural Network[J] Modular Machine Tool \& Automatic Manufacturing Technique, 2015(4):74-77

5 ChenFa Fa,Tang Bao Ping,Huang Qing Qing.Rotating machinery fault diagnosis based on Elman neural network optimized by immune genetic algorithm[J]Journal of Chong qing University,2012, 35(5):7-13 
6 Wang Xiao xia,Ma Liang yu,Wang Bing shu. Application of evolutionary Elman neural network in real-time data forecasting $[\mathrm{J}]$ Electric Power Automation Equipment, 2011,31(12):77-81

7 Liu J X, Wang F, Xie K M. Application of improved mind evolutio nary algorithm in wide band impendence transformer design[C]Procofthe 4th Int Confon Natural Com putation (ICNC. 08). Ji.nan, 2008: 428-432

8 WANG Fang,XIE Ke-ming,LIU Jian-xia. Swarm intelligence based MEA design[J]Control and Decision, 2010,25(1) : 145-148

9 Huang Jun,Feng Yong Jian.Improved Pressure Measurement Precision for Vacuum Insulation Panel with Mind Evolutionary Algorithm[J]Chinese Journal of Vacuum Science and Technology, 2015,35(5) : 528-532 10 Li Xiu Guang.Fault Diagnosis for Power Transformer Based on Mind Evolution Algorithm Optimized Neural Network[D]Taiyuan University of Technology,2010: 6669 